\title{
Conditions on Periodicity for Sum-Free Sets
}

\author{
Neil J. Calkin and Steven R. Finch
}

\section{CONTENTS}

1. Introduction

2. Definitions

3. The Bijection Between Binary Sequences and Sum-Free Sets

4. Periodicity of Sum-Free Sets

5. Computational Evidence

References
Cameron has introduced a natural one-to-one correspondence between infinite binary sequences and sets of positive integers with the property that no two elements add up to a third. He observed that, if a sum-free set is ultimately periodic, so is the corresponding binary sequence, and asked if the converse also holds. We present here necessary and sufficient conditions for a sum-free set to be ultimately periodic, and show how these conditions can be used to test specific sets. These tests produce the first evidence of a positive nature that certain sets are, in fact, not ultimately periodic.

\section{INTRODUCTION}

Infinite binary sequences are in natural one-to-one correspondence with sum-free sets of positive integers, that is, sets of which no element is the sum of two elements (Section 3). Cameron [1987] observed that, if a sum-free set is ultimately periodic, the corresponding binary sequence is ultimately periodic, and asked whether the converse is also true. This question is still open, but there is some indication that the answer is no: Some apparently aperiodic sets correspond to ultimately periodic binary sequences. Although some of these sets are relatively simple, a proof of their aperiodicity has been elusive, because no method is known that will show that a sum-free set is not ultimately periodic from a consideration of only finitely many elements.

In this work (Section 4) we introduce two new functions $g$ and $\bar{g}$, defined on the positive integers, and we show that the behavior of these functions determines whether a set is ultimately periodic or not. More precisely, we prove that, if its corresponding binary sequence is ultimately periodic, a sum-free set is ultimately periodic if and only if $g$ is bounded, and that if $g$ is not bounded, $\bar{g}(n)$ grows at least as fast as $\log n$. 
In Section 5 we summarize the results of our systematic tests of periodicity over large classes of sum-free sets.

\section{DEFINITIONS}

Let $S$ be a sum-free set. This means that $S$ is a subset of $\mathbb{N}$ (the set of positive integers) and that there are no $x, y, z \in S$ with $x+y=z$. We do not require $x$ and $y$ to be distinct. We denote the set of sum-free sets of positive integers by $\mathcal{S}$.

$S$ is ultimately complete if, for all sufficiently large $n$, either $n \in S$ or there exist $x, y \in S$ such that $x+y=n$.

$S$ is periodic if there exists a positive integer $m$ such that, for all $n \geq 1$, we have $n \in S$ if and only if $n+m \in S$.

$S$ is ultimately periodic if there exist positive integers $m, n_{0}$ such that, for all $n \geq n_{0}$, we have $n \in S$ if and only if $n+m \in S$. In this case we call $n_{0}$ a preperiod and $m$ a period of $S$. There is a unique minimum period, since the greatest common divisor of two periods is also a period, but we usually won't insist that either $m$ or $n_{0}$ be minimal. For fixed $m$ and $n_{0}$ we set

$$
\begin{aligned}
S_{0} & =S \cap\left\{1, \ldots, n_{0}-1\right\}, \\
S_{\text {per }} & =S \cap\left\{n_{0}, n_{0}+1, \ldots\right\}, \\
\bar{S} & =S_{\text {per }} \bmod m .
\end{aligned}
$$

Then $S=S_{0} \cup S_{\text {per }}$ and

$$
S_{\text {per }}=\left\{n \in \mathbb{N}: n \geq n_{0} \text { and } n \bmod m \in \bar{S}\right\} .
$$

For example, if $S$ is the set of odd positive integers, which is periodic, we have $\bar{S}=\{1\} \bmod 2$. Removing a finite number of elements from $S$ makes it only ultimately periodic, but does not change $\bar{S}$. For the ultimately periodic set $S=\{1\} \cup\{3 n+2$ : $n \in \mathbb{N}\}$ we have $\bar{S}=\{2\} \bmod 3$, and so on.

\section{THE BIJECTION BETWEEN BINARY SEQUENCES AND SUM-FREE SETS}

Let $\sigma$ be an element of $2^{\mathbb{N}}$, say $\sigma_{1} \sigma_{2} \sigma_{3} \ldots$, where $\sigma_{i} \in\{0,1\}$ for every $i$. We construct the sum-free set $S$ associated to $\sigma$ by testing one integer $n$ at a time, in increasing order, for the possibility of inclusion in $S$. If $n$ is a sum of integers already in $S$, it obviously should not be included. Otherwise, the next element of $\sigma$ dictates whether or not $n$ is included in $S$.

Formally, we define sets $S_{i}$ and $U_{i}$ inductively, starting with $S_{0}=U_{0}=\varnothing$. Let $n_{i}$ be the least element of $\mathbb{N}$ that is not in $S_{i-1} \cup\left(S_{i-1}+S_{i-1}\right) \cup U_{i-1}$, where $A+B=\{a+b: a \in A, b \in B\}$. Then define

$$
\begin{gathered}
S_{i}= \begin{cases}S_{i-1} \cup\left\{n_{i}\right\} & \text { if } \sigma_{i}=1, \\
S_{i-1} & \text { if } \sigma_{i}=0 ;\end{cases} \\
U_{i}= \begin{cases}U_{i-1} & \text { if } \sigma_{i}=1, \\
U_{i-1} \cup\left\{n_{i}\right\} & \text { if } \sigma_{i}=0 .\end{cases}
\end{gathered}
$$

Let $S=\bigcup_{i} S_{i}$; then, since each $S_{i}$ is sum-free, and since $S_{i} \subset S_{i+1}$, the union $S$ is also sum-free.

We write $S=\theta(\sigma)$, defining a map $\theta: 2^{\mathbb{N}} \rightarrow \mathcal{S}$. For example,

$$
\begin{aligned}
& \theta(1111111111 \ldots)=\{1,3,5,7,9,11,13,15, \ldots\}, \\
& \theta(0101010101 \ldots)=\{2,5,8,11, \ldots\} \\
& \theta(1010101010 \ldots)=\{1,4,7,10, \ldots\} \\
& \theta(1010010101 \ldots)=\{1,4,8,11,14, \ldots\} .
\end{aligned}
$$

This map is a bijection. Indeed, if $S$ is a sum-free set, we define a ternary sequence $\tau$ by setting

$$
\tau_{n}= \begin{cases}1 & \text { if } n \in S \\ * & \text { if } n \in S+S, \\ 0 & \text { otherwise }\end{cases}
$$

then convert this to a binary sequence $\sigma$ by deleting all *'s. It is an easy exercise to check that this correspondence is inverse to $\theta$. We formalize the $*$ erasing procedure since the notation will be useful later: Let $\nu$ be the unique increasing bijection from $\mathbb{N}$ onto the set $\mathbb{N} \backslash(S+S)=\tau^{-1}(\{0,1\})$; then $\sigma_{n}=\tau_{\nu(n)}$.

The bijection $\theta: 2^{\mathbb{N}} \rightarrow \mathcal{S}$ is actually a homeomorphism if $2^{\mathbb{N}}$ is given the dyadic metric (two sequences are at distance $2^{-k}$ if they differ for the first time at the $(k+1)$-st place) and $\mathcal{S}$ is given the analogous metric (the distance between $S_{1}$ and $S_{2}$ 
is $2^{-k}$ if $k+1$ is the least element in the symmetric difference $S_{1} \triangle S_{2}$ ).

We will see in Lemma 4.3 that, if $S$ is ultimately periodic, so is $\theta^{-1}(S)$.

Proposition 3.1. $S$ is ultimately complete if and only if $\theta^{-1}(S)$ contains only finitely many zeros.

Proof. In the construction of $\theta(\sigma)$, an element is not included if and only if either it is a sum of smaller elements already in the set, or the corresponding term in the binary sequence is zero. Thus, if $S$ is ultimately complete, we can only have finitely many elements excluded because of zeros in $\theta^{-1}(S)$.

This implies that the set of ultimately complete sum-free sets is countable. By contrast:

Proposition 3.2. There are uncountably many maximal sum-free sets.

Naturally, a sum-free set is maximal if it cannot be enlarged without destroying the sum-free property.

Proof. Consider the set $\{9,11,14,16,19,21, \ldots\}=$ $\{5 k \pm 1: k=2,3, \ldots\}$, which is clearly sum-free. If we add to this set the element 2 , we find that the only solutions to the equation $x+y=z$ are of the form $5 k+1=(5 k-1)+2$. Now consider an arbitrary partition of $\{2,3,4,5, \ldots\}$ into two parts $N_{1}$ and $N_{2}$, and define

$S_{N_{1}, N_{2}}=\{2\} \cup\left\{5 k-1: k \in N_{1}\right\} \cup\left\{5 k+1: k \in N_{2}\right\}$.

This set is sum-free, since by definition $N_{1} \cap N_{2}=$ $\varnothing$. Then no integer of the form $5 k-1$, for $k \in N_{2}$, or of the form $5 k+1$, for $k \in N_{1}$, can be adjoined to $S_{N_{1}, N_{2}}$, since such integers are differences or sums of pairs of elements in $S_{N_{1}, N_{2}}$. Now extend $S_{N_{1}, N_{2}}$ to a maximal sum-free set $T_{N_{1}, N_{2}}$, using Zorn's lemma. By the preceding comments, $T_{N_{1}, N_{2}}$ and $T_{M_{1}, M_{2}}$ are distinct if $N_{1} \neq M_{1}$. Since there are uncountably many partitions of $\{2,3,4, \ldots\}$, we have proved the proposition.

Since the lower asymptotic density of $T_{N_{1}, N_{2}}$ is at least $\frac{1}{5}$, we get the following result, which answers a question of Stewart (personal communication).
Corollary 3.3. There are uncountably many aperiodic maximal sum-free sets of positive lower density.

\section{PERIODICITY OF SUM-FREE SETS}

We shall now consider one of the most intriguing questions regarding sum-free sets, namely the relationship between the periodicity of a binary string $\sigma$ and that of the associated sum-free set $\theta(\sigma)$. Cameron (personal communication) has asked if either of these statements is true:

Conjecture 4.1. A binary string $\sigma$ is ultimately periodic if and only if $\theta(\sigma)$ is ultimately periodic.

Conjecture 4.2. A binary string $\sigma$ has only finitely many zeros if and only if $\theta(\sigma)$ is ultimately periodic and ultimately complete (by Proposition 3.1, this is the same as saying that any ultimately complete sum-free set is ultimately periodic).

Clearly 4.1 implies 4.2 , but not necessarily vice versa. Lemma 4.3 below shows that the "if" part of the first conjecture holds, and that of the second follows of course from Proposition 3.1. The converses are still open; however, since the questions were first posed, we have found evidence to suggest that Conjecture 4.1 is false, and Cameron [1987] has found evidence that 4.2 may also be false.

Lemma 4.3 [Cameron 1987]. If $S=\theta(\sigma)$ is ultimately periodic, so is $\sigma$.

Proof. Let $n_{0}$ be a preperiod and $m$ a period of $S$. Consider the ternary sequence $\tau$ associated with $S$ via (3.1). For $n \geq 2 n_{0}$, we have $\tau_{n}=1 \Longleftrightarrow n \in S$ $\Longleftrightarrow n \in S_{\mathrm{per}} \Longleftrightarrow n \bmod m \in \bar{S}$ by (2.1), and also

$$
\begin{aligned}
\tau_{n}=* & \Longleftrightarrow n \in S+S \\
& \Longleftrightarrow n \in\left(S_{\mathrm{per}}+S_{\mathrm{per}}\right) \cup\left(S_{\mathrm{per}}+S_{0}\right) \\
& \Longleftrightarrow n \bmod m \in(\bar{S}+\bar{S}) \cup\left(\bar{S}+\left(S_{0} \bmod m\right)\right)
\end{aligned}
$$

because $n$ is too large to be in $S_{0}+S_{0}$. Thus $\tau_{n}$ depends solely upon the congruence class of $n$ $\bmod m$. This shows that $\tau$ is ultimately periodic, and therefore so is $\sigma$. A period of $\sigma$ is given by $\nu^{-1}(n+m)-\nu^{-1}(n)$, where $n \in S$ exceeds $2 n_{0}$ (the map $\nu$ is defined after (3.1)). 
Where will we run into difficulties when we try to reverse this proof? The crucial step involves the erasing of the $*$ 's in $\tau$ : given a periodic sequence $\sigma$ it is easy to insert $*$ 's in such a way that the resulting ternary sequence is aperiodic (for example, insert a $*$ after every $p_{k}$-th 1 , where $p_{k}$ is the $k$-th prime). Of course, it is unlikely that such insertions would leave a sum-free set: Conjecture 4.1 states essentially that only by inserting in a periodic manner is it possible to ensure that $S$ is sumfree.

In trying to prove the "only if" part of the conjectures, one might be helped by a sufficient criterion that ensures that a sum-free set is ultimately periodic. The following lemma is one such criterion: it says that if a sum-free set $S$ is ultimately periodic, this fact can be proved by considering only $\theta^{-1}(S)$ and a finite prefix of $S$.

Lemma 4.4. Let $S$ be a sum-free set, with associated binary sequence $\sigma$, and let $m$ be an integer. Set

$$
S_{k}=S \cap\{k m+1, k m+2, \ldots,(k+1) m\}
$$

for $k=1,2,3$, and $t_{k}=\nu^{-1}\left(\max S_{k}\right)$. Suppose that $S_{3}=S_{2}+m=S_{1}+2 m$, and that $\sigma_{t+t_{2}}=\sigma_{t+t_{3}}$ for all $t>0$ - in particular, $\sigma$ is ultimately periodic of period $p=t_{3}-t_{2}$. Then $S$ is ultimately periodic of period $m$ and preperiod $m$.

Proof. We show by induction that $n \geq m$ is in $S$ if and only if $n+m$ is in $S$. This is true of $n=m+1$, $\ldots, 3 m$ because $S_{2}$ and $S_{3}$ are translates of $S_{1}$. Therefore we can take $n>3 m$, and assume that the claim is true for all lesser values of $n(\geq m)$.

In fact we will show that $\tau_{n+m}=\tau_{n}$. Take first the case $\tau_{n}=*$, that is, $n \notin S+S$. Then also $n+m \notin S+S$; otherwise, express $n+m$ as $x+y$ with $x, y \in S$ and $x \in\{2 m+1, \ldots, n-1\}$ (using the fact that $n>3 m$ ), and apply the induction assumption to write $n=(x-m)+y \in S+S$. Analogously, $n+m \notin S+S$ implies $n \notin S+S$; otherwise, set $n=x+y$ with $x, y \in S$ and $x \in\{m+1, \ldots, n-1\}$, and apply the induction assumption. This shows that $\tau_{n+m}=* \Longleftrightarrow \tau_{n}=*$.
On the other hand, if $\tau_{n} \neq *$ and $\tau_{n+m} \neq *$, we have $\nu^{-1}(n+m)=\nu^{-1}(n)+p$ (by induction; the base case is $n=\max S_{2}$ and $n+m=\max S_{3}$, and $n$ is in the image of $\nu$ if and only $n+m$ is). But then $\tau_{n+m}=\sigma_{\nu^{-1}(n+m)}=\sigma_{\nu^{-1}(n)+p}=\sigma_{\nu^{-1}(n)}=\tau_{n}$, where the second-to-last equality comes from the lemma's assumption.

In order to test Cameron's conjectures, we generated the sum-free sets corresponding to periodic binary inputs with period at most seven. For all inputs with periods of length at most four, the corresponding sum-free set was ultimately periodic, with a small preperiod (usually fewer than 10) and a small period (always less than 25). Of the thirty inputs with periods of length five, all but three (ن்100i, $\dot{0} 101 \dot{0}, 1001 \dot{0})$ gave sum-free sets that were quickly periodic. The set

$\theta(\dot{0} 100 \dot{1})=$

$$
\{2,6,9,14,19,26,29,36,39,47,54,64,69,79,84,91, \ldots\},
$$

certainly appears to be aperiodic: the sequence of differences between consecutive elements up to $10^{7}$ exhibits long strings that are repeated, separated by short "glitches" that show no sign of settling down to be periodic. Other potential counterexamples to Cameron's conjecture will be exhibited in Section 5.

This, of course, is all evidence of a rather flimsy type: "We looked, but we couldn't find anything". We shall now state theorems that lead to more positive evidence that certain sum-free sets, $\theta(\dot{0} 100 \dot{1})$ among them, are aperiodic.

Define functions $g$ and $\bar{g}$ on $\mathbb{N}$ by

$$
\begin{aligned}
& g(n)=\left\{\begin{array}{l}
0 \quad \text { if } n \notin S+S, \\
\min \{x \in S: x+y=n \text { for some } y \in S\} \\
\text { otherwise }
\end{array}\right. \\
& \bar{g}(n)=\max _{k \leq n} g(k) .
\end{aligned}
$$

Theorem 4.5. $S$ is ultimately periodic if and only if $\sigma$ is ultimately periodic and $\bar{g}$ is ultimately constant (that is, $g$ is bounded). 
Proof. For $S$ ultimately periodic, with preperiod $n_{0}$ and period $m$, we get $g(n)<n_{0}+m$ using the equivalence $\tau_{n}=* \Longleftrightarrow n \in\left(S_{\text {per }}+S_{\text {per }}\right) \cup\left(S_{\text {per }}+S_{0}\right)$ from the proof of Lemma 4.3 (for $n \in S_{\text {per }}+S_{\text {per }}$, one of the summands can be taken less than $n_{0}+m$, by periodicity).

Conversely, suppose $\sigma=\theta^{-1}(S)$ is ultimately periodic of period $p$, and take $r$ large enough that $\nu^{-1}(r)$ is in the periodic part of $\sigma$. Suppose also that $g(n) \leq k$ for all $n$. Define $S_{n}=S \cap\{n+1$, $n+2, \ldots, n+k\}$ for $n \geq 0$, so that $S+S=S_{0}+S$. Then, for $n>r$, the question whether $n+k+1$ belongs to $S$, and thus to $S_{n+1}$, depends only on $S_{0}, S_{n}$, and $\sigma\left(j_{n}\right)$, where $j_{n}=\nu^{-1}\left(\max S_{n}\right)$; also $\sigma\left(j_{n}\right)$ depends only on $j_{n} \bmod p$. Setting $T_{n}=$ $S_{n}-n$ and $i_{n}=j_{n} \bmod p$, it follows that $T_{n+1}$ and $i_{n+1}$ are determined by $T_{n}$ and $i_{n}$ (the dependence being controlled by $S_{0}$ and $\sigma$, which are fixed). $T_{n}$ has at most $2^{k}$ values as $n$ varies, and $i_{n}$ has at most $p$ values, so there exist $n_{0}$ and $n_{0}+m$, both in the interval $\left\{r, r+1, \ldots, r+2^{k} p\right\}$, such that $T_{n_{0}}=T_{n_{0}+m}$ and $i_{n_{0}}=i_{n_{0}+m}$. Thereafter, $T_{n}$ and $T_{n+m}$ coincide. This proves that $S$ is ultimately periodic with preperiod $n_{0}$ and period $m$.

In fact, Theorem 4.5 can be strengthened: for a sum-free set that is not ultimately periodic, $\bar{g}$ must grow at least logarithmically, as we now show.
Theorem 4.6. Suppose $\sigma=\theta^{-1}(S)$ is ultimately periodic, with period $p$, and take $r>2 \min S$ large enough that $\nu^{-1}(r)$ is in the periodic part of $\sigma$. If there exists $N>4 r$ such that

$$
\bar{g}(N)<\log _{2} \frac{N-4 r}{4 p},
$$

then $S$ is ultimately periodic.

Proof. Let $N>4 r$ satisfy (4.1), and set $k=\bar{g}(N)$ : thus $N \geq 4(r+2 k p)$. Then, copying the notation and reasoning from the proof of Theorem 4.5, we can find $n_{0}$ and $n_{0}+m$, both in the interval $\left\{r, r+1, \ldots, r+2^{k} p\right\}$, such that $T_{n_{0}}=T_{n_{0}+m}$ and $i_{n_{0}}=i_{n_{0}+m}$. Thereafter, $T_{n}$ and $T_{n+m}$ coincide at least until $n+m=N$. Replace $m$ by the least multiple of $m$ greater than $n_{0}$; this number is still bounded by $r+2^{k} p \leq \frac{1}{4} N$. But now $m$ satisfies the conditions of Lemma 4.4 , proving that $S$ is ultimately periodic.

Computing the values of $\bar{g}(n)$ for the set $\theta(\dot{0} 100 \dot{1})$, for all $n \leq 200000$, we find that $\bar{g}$ appears to be very far from bounded: in fact it seems to increase in a roughly linear fashion, throughout the whole range $n<10^{7}$. See Table 1 .

If it could be shown for such a set $S$ that such behavior continues, namely that there exist an infinite number of $n$ such that $g(n) / n$ is close to $\frac{1}{2}$,

\begin{tabular}{|rr|rc|rc|rc|cc|cc|cc|}
\hline$n$ & $\bar{g}(n)$ & $n$ & $\bar{g}(n)$ & $n$ & $\bar{g}(n)$ & $n$ & $\bar{g}(n)$ & $n$ & $\bar{g}(n)$ & $n$ & $\bar{g}(n)$ & $n$ & $\bar{g}(n)$ \\
\hline 4 & 2 & 242 & 121 & 1820 & 597 & 4632 & 2068 & 14779 & 7104 & 47437 & 23304 & 121318 & 57969 \\
12 & 6 & 274 & 137 & 1850 & 627 & 4945 & 2381 & 16129 & 7675 & 49313 & 24133 & 126698 & 63349 \\
18 & 9 & 322 & 161 & 2028 & 805 & 5128 & 2564 & 19678 & 9839 & 50678 & 25180 & 137806 & 65796 \\
33 & 14 & 348 & 174 & 2058 & 835 & 6053 & 2676 & 22914 & 11457 & 50996 & 25498 & 142928 & 71464 \\
52 & 26 & 362 & 181 & 2103 & 880 & 6411 & 3034 & 24624 & 12312 & 65250 & 28709 & 171101 & 81091 \\
72 & 36 & 637 & 237 & 2356 & 1133 & 6674 & 3297 & 27324 & 13394 & 68410 & 30974 & 188656 & 82178 \\
94 & 47 & 647 & 247 & 2371 & 1148 & 6709 & 3332 & 30140 & 14127 & 75499 & 37613 & 199466 & 99733 \\
133 & 54 & 690 & 345 & 2401 & 1178 & 6754 & 3377 & 40677 & 15179 & 82800 & 38422 & $\ldots \ldots \ldots \ldots$ \\
182 & 91 & 885 & 430 & 2446 & 1223 & 10360 & 4014 & 43908 & 16281 & 88756 & 44378 & 605846 & 1211692 \\
192 & 96 & 1288 & 445 & 3650 & 1522 & 11144 & 4798 & 43948 & 21974 & 111332 & 54455 & $\ldots \ldots \ldots \ldots \ldots$ \\
227 & 106 & 1457 & 577 & 4394 & 1795 & 12692 & 6346 & 46355 & 22222 & 112419 & 55542 & 4621889 & 9662060 \\
\hline
\end{tabular}

TABLE 1. For $S=\theta(\dot{0} 1001)$, the table gives the points $n \leq 200000$ at which $\bar{g}(n)$ has just increased, and the corresponding values $\bar{g}(n)$. Also given are the largest $n<10^{7}$ for which $g(n)=\frac{1}{2} n$ (penultimate entry), and the largest $n<10^{7}$ for which $\bar{g}$ increases (last entry). 
say, it would follow immediately from Theorem 4.5 that $S$ is aperiodic; it does not, however, appear that it is a simple matter to prove this.

\section{COMPUTATIONAL EVIDENCE}

If we could prove $\theta(\dot{0} 100 \dot{i})$ is aperiodic, there would be no need to list further potential counterexamples to Cameron's conjecture. Since we couldn't, we found it to be of some value to test periodicity over large classes of sum-free sets, in the hope that a recognizable pattern to the counterexamples might eventually emerge. Table 2 summarizes the possible counterexamples we have found among all periodic binary sequences $\sigma$ of periods 5,6 or 7 . This includes the three potential counterexamples mentioned earlier.

\begin{tabular}{|llll|}
\hline$\dot{0} 100 \dot{1}$ & $\dot{0} 1000 \dot{1}$ & $\dot{0} 01000 \dot{1}$ & $\dot{0} 10101 \dot{1}$ \\
$\dot{0} 101 \dot{0}$ & $\dot{0} 1100 \dot{1}$ & $\dot{0} 01001 \dot{0}$ & $\dot{0} 10110 \dot{1}$ \\
$\dot{1} 001 \dot{0}$ & $\dot{0} 1110 \dot{0}$ & $\dot{0} 10000 \dot{1}$ & $\dot{0} 11000 \dot{1}$ \\
& $\dot{1} 0001 \dot{0}$ & $\dot{0} 10001 \dot{0}$ & $\dot{1} 00001 \dot{0}$ \\
& $\dot{10100 \dot{1}}$ & $\dot{0} 10010 \dot{0}$ & $\dot{100010 \dot{0}}$ \\
& $\dot{1} 0101 \dot{1}$ & $\dot{0} 10010 \dot{1}$ & $\dot{1} 00011 \dot{0}$ \\
& & $\dot{0} 10101 \dot{0}$ & $\dot{101010 \dot{0}}$ \\
\hline
\end{tabular}

TABLE 2. Periodic binary sequences whose associated sum-free sets are incomplete and appear to be aperiodic (aperiodicity checked up to $10^{7}$ ).

We note that periodicity in sum-free sets need not arrive quickly. $S=\theta(\dot{0} 11001 \dot{1})$ has minimal period $m=10710$, after a transient phase of approximately 89000 terms. Moreover, the largest integer $n \in S$ for which $n+m \notin S$ is $n=489115$, and the largest integer $n \notin S$ for which $n+m \in S$ is $n=489108$.

In addition to periodic binary sequences of periods up to 7 , we studied those having period 3 and preperiod 2 (that is, of the form $u v \dot{x} y \dot{z}$ ), and those having period 2 and preperiod 5 . The potentially aperiodic sum-free sets among them (also checked up to $\left.10^{7}\right)$ are $\theta(00 \dot{0} 0 \dot{1}), \theta(00001 \dot{1} \dot{0}), \theta(11000 \dot{0} \dot{i})$, and $\theta(001100 \dot{1})$. These are the simplest such cases, that is, the binary inputs simultaneously have minimal preperiod and minimal period.
Cameron [1987] found the first potentially aperiodic complete sum-free set; it is entry 1 in Table 4 (the notation will be explained shortly). The existence of such a set suggests that Dickson's problem [Dickson 1934; Guy 1980, Problem E32] may have a negative solution. Queneau [1972] and Finch [1992] have studied a variation of this problem involving what are known as 0-additive sequences; an update on this direction of research appears in [Guy 1993].

By the base of an ultimately complete sum-free set $S=\left\{s_{1}<s_{2}<\cdots<s_{n}<\cdots\right\}$ we mean the minimal set of $S$-elements $B=\left\{s_{1}, s_{2}, \ldots, s_{n}\right\}$ such that recursive application of the greedy algorithm, starting with $B$, gives the sum-free set $S$.

By the phrase "all sum-free bases up to $p$ " we mean the collection of all sets $B$ that are bases of ultimately complete sum-free sets $S$ and whose largest element is at most $p$. For example, the sumfree bases up to 7 are

$$
\begin{aligned}
& \{1\},\{2\},\{3\},\{4\},\{5\},\{6\},\{7\}, \\
& \{1,4\},\{1,5\},\{1,6\},\{1,7\},\{2,5\},\{2,6\},\{2,7\}, \\
& \{3,5\},\{3,7\},\{4,6\},\{4,7\},\{5,7\}, \\
& \{1,3,7\},\{1,4,7\},\{4,5,7\} .
\end{aligned}
$$

We examined each of the 76080 sum-free bases up to 27 and determined whether each of the corresponding complete sum-free sets were periodic (checked up to $10^{7}$ ). We did the same for all sumfree bases up to 35 with three or fewer elements. All apparently aperiodic cases (for which $g$ appears to be unbounded and no pattern is seen) are listed in Table 3. Table 4, by contrast, lists those cases that we classify as tentatively periodic. Entry 1 in this table is Cameron's example. Entry 6 is the same, minus one term, as $\{15,16,18,21,22,24,27\}$, which is not listed to avoid duplication. Entry 7 is unexpected: the maximum $g$-value is quite small, but no clear signs of periodicity are apparent.

We stress that periodicity need not arrive quickly. For example, the periodic complete sum-free set $S$ based on $\{10,14,15,17,22\}$ has minimal period $m=2875722$ after a transient phase of approximately 584000 terms. The largest integer $n \in S$ for 


\begin{tabular}{|ll|}
\hline$\{8,18,30\}$ & $\{1,3,8,20,26\}$ \\
$\{8,27,32\}$ & $\{2,15,16,23,27\}$ \\
$\{9,16,29\}$ & $\{5,6,14,23,27\}$ \\
$\{9,26,32\}$ & $\{3,12,17,19,21,27\}$ \\
$\{9,28,35\}$ & $\{10,13,15,16,17,24\}$ \\
$\{10,18,34\}$ & $\{10,15,16,18,22,27\}$ \\
$\{11,26,35\}$ & $\{12,15,17,18,19,25\}$ \\
$\{12,21,35\}$ & $\{14,16,17,18,21,27\}$ \\
$\{9,21,24,27\}$ & $\{6,14,17,18,22,25,27\}$ \\
$\{11,16,17,26\}$ & \\
\hline
\end{tabular}

TABLE 3. Apparently aperiodic complete sum-free sets listed by base (checked up to $10^{7}$ ).

\begin{tabular}{|clrc|}
\hline$\#$ & \multicolumn{1}{c}{ base } & $\bar{g}\left(10^{7}\right)$ & est. per. \\
\hline 1 & $\{3,4,13,18,24\}$ & 2937317 & 3274006 \\
2 & $\{8,14,15,17,26\}$ & 2898098 & $?$ \\
3 & $\{14,15,16,18,21,26\}$ & 1349528 & $?$ \\
4 & $\{14,15,18,20,22,24,26\}$ & 1424518 & 1291498 \\
5 & $\{4,17,18,19,24,27\}$ & 3132839 & 1022104 \\
6 & $\{15,16,18,22,24,27\}$ & 2330099 & 2673770 \\
7 & $\{4,21,32\}$ & 770538 & $?$ \\
\hline
\end{tabular}

TABLE 4. Tentatively periodic complete sum-free sets listed by base $\left(\bar{g}\left(10^{7}\right) \leq 3.2 \cdot 10^{6}\right)$. The last column gives our best estimate of the period. which $n+m \notin S$ is $n=4562648$, and the largest integer $n \notin S$ for which $n+m \in S$ is $n=4453256$.

\section{REFERENCES}

[Cameron 1987] P. J. Cameron, "Portrait of a typical sum-free set", pp. 13-42 in Surveys in Combinatorics, 1987 (edited by C. Whitehead), London Math. Soc. Lecture Notes 123, Cambridge U. Press, Cambridge, 1987.

[Dickson 1934] L. E. Dickson, "The converse of Waring's problem". Bull. Amer. Math. Soc. 40 (1934), 711-714.

[Finch 1992] Steven R. Finch, "Are 0-additive sequences always regular?", Amer. Math. Monthly 99 (1992), 671-673.

[Guy 1980] Richard K. Guy, Unsolved Problems in Number Theory, Springer, New York, 1980.

[Guy 1993] Richard K. Guy, "A quarter century of Monthly unsolved problems, 1969-1993", Amer. Math. Monthly 100 (1993), 945-949.

[Queneau 1972] Raymond Queneau, "Sur les suites $s$ additives", J. Combin. Theory A12 (1972), 31-71. English summary in Math. Rev. 46, 1741.

Neil J. Calkin, School of Mathematics, Georgia Institute of Technology, Atlanta, GA 30332

(calkin@math.gatech.edu)

Steven R. Finch, 6 Foster Street, Wakefield, MA 01880 (sfinch@gnu.ai.mit.edu)

Received April 20, 1995; accepted in revised form November 20 